\title{
AVALIAÇÃO DA ADESÃO INTERFACIAL DAS FIBRAS DE COCO E PALF NA MATRIZ EPÓXI POR ENSAIO PULLOUT*
}

Fernanda Santos da Luz ${ }^{1}$ Sergio Neves Monteiro ${ }^{2}$

\section{Resumo}

Nas últimas décadas, houve um aumento da aplicação de fibras naturais lignocelulósicas (FNLs) como reforços de compósitos poliméricos em diversos setores industriais, principalmente no segmento automotivo. Esse crescente interesse em FNLs se deve não somente aos benefícios ambientais, mas também às vantagens técnicas, econômicas e sociais. Dentre as FNLs, destacam-se as fibras de coco e as fibras da folha do abacaxizeiro (PALF) que são produtos residuais do cultivo de suas respectivas frutas e, por isso, possuem baixo custo e são abundantemente disponíveis. As características físicas dessas fibras se diferem bastante, sendo o PALF caracterizado por suas fibras longas e diâmetro equivalente relativamente baixo. O presente trabalho avaliou o desempenho das fibras de coco e PALF em testes de pullout com o objetivo de caracterizar o comprimento crítico e a adesão interfacial dessas fibras na matriz de epóxi. Os resultados mostraram um comprimento crítico $65 \%$ maior para a fibra de coco em relação ao PALF, o que indica maior adesão interfacial do PALF com a matriz epóxi e pode ser atribuída a superfície naturalmente mais rugosa do PALF.

Palavras-chave: Teste de Pullout; Fibras naturais lignocelulósicas; Comprimento crítico; Adesão interfacial.

\section{EVALUATION OF INTERFACIAL ADHESION OF THE COIR AND PALF FIBERS IN THE EPOXY MATRIX BY PULLOUT TEST}

\section{Abstract}

Over recent decades, the application of natural lignocellulosic fibers (NLFs) as reinforcements in polymeric composites was increased in several industrial sectors, mainly in automotive segment. This growing interest in NLFs is due not only to environmental benefits but also to technical, economic and social advantages. Among the NLFs, the coir fibers and pineapple leaves fibers (PALF) are byproducts of their fruits cultivation, therefore, these are inexpensive and abundantly available. The physical characteristics of these fibers differ greatly, the PALF are long fibers with relatively low equivalent diameter unlike coir fiber. The present work evaluated the performance of PALF and coir fibers in pullout tests to determine the critical length and the interfacial adhesion of these fibers with epoxy matrix. The results showed a critical length $65 \%$ higher for the coir fiber in comparison to PALF, which indicates greater interfacial adhesion of PALF with epoxy matrix and may be attributed to the naturally rougher surface of PALF.

Keywords: Pullout test; Natural lignocellulosic fibers; Critical length; Interface adhesion.

1 Engenheira de Materiais, M.C, Doutoranda em Ciência dos Materiais, Seção de Engenharia Mecânica e de Materiais, Instituto Militar de Engenharia, Rio de Janeiro, RJ, Brasil. fsl.santos@gmail.com

2 Engenheiro Metalurgista, Ph.D, Professor Titular, Seção de Engenharia Mecânica e de Materiais, Instituto Militar de Engenharia, Rio de Janeiro, RJ, Brasil. 


\section{INTRODUÇÃO}

As fibras naturais lignocelulósicas (FNLs) vêm conquistando espaço no mercado e têm sido utilizadas como uma alternativa às fibras sintéticas empregadas como reforços em materiais compósitos. Um exemplo dessa tendência é a aplicação das FNLs em componentes internos na indústria automotiva [1-5]. Alguns compósitos termoplásticos reforçados com fibras lignocelulósicas já vêm sendo utilizados também nas indústrias de móveis, embalagens e construção [1-3].

As FNLs possuem diversas vantagens, tais como, baixo custo, baixa densidade, natureza biodegradável e o fato de serem provenientes de fontes renováveis. Outro aspecto que diferencia a FNL da fibra sintética é o caráter hidrofílico da primeira, dado pela presença dos grupos hidroxilas em sua composição [6,7]. Entretanto, os polímeros comumente utilizados em compósitos possuem caráter hidrofóbico e a umidade na superfície da fibra age como um separador na interface fibra/matriz. Para melhorar a adesão interfacial as FNLs podem passar por pré-tratamentos de modificação química de superfície ou de secagem, essas e outras técnicas têm sido reladas na literatura $[2,6,8]$.

De modo geral, a falha em compósitos poliméricos reforçados com fibras ocorre por uma combinação de mecanismos. Esses mecanismos dependem de uma série de fatores tais como, porcentagem de fibra usada como reforço, propriedades mecânicas da fibra, adesão interfacial entre fibra e matriz, entre outros. Dentre esses mecanismos pode-se citar o arrancamento da fibra (pullout), observado em compósitos com matriz de elevada resistência reforçado com fibras frágeis, as quais se rompem e geram uma concentração de tensões, que é aliviada após o desprendimento da fibra/matriz, sendo a sua principal causa a baixa adesão interfacial $[9,10]$.

Por isso, uma forma de avaliar a adesão interfacial entre a fibra e a matriz é por meio do ensaio de pullout proposto inicialmente por Kelly e Tyson [11] e adaptado posteriormente por Monteiro e D'Almeida [12] para as fibras lignocelulósicas em particular. Através desse ensaio é possível determinar o comprimento crítico da fibra e o valor da tensão cisalhante interfacial o qual está associado à eficiência da ligação existente entre a matriz e a superfície da fibra, pois essa caracteriza o grau de acoplamento das fases no compósito [12].

No presente trabalho foram avaliadas as fibras de coco e PALF em uma matriz epoxídica. Embora a fibra de coco, rica em lignina e com elevado ângulo microfibrilar [8], seja fraca comparada com as fibras ricas em celulose tais como, sisal, juta, PALF, certas propriedades únicas tornam-na atrativa como reforço em compósitos poliméricos. As fibras de coco possuem uma elevada deformação até a ruptura de 15 a 40\%, resistência à degradação microbiana e por fungos, maior resistência à umidade e capacidade de suportar calor e salinidade [13-17]. Essa fibra possui uma fina camada superficial alifática (camada de cera) que consiste em uma cadeia longa de ácidos graxos e seus produtos de condensação, a qual é incompatível com as resinas poliéster e a remoção da mesma se faz necessária para uma boa adesão interfacial em tais compósitos de matriz polar, como relatado em alguns estudos [18]. Além disso, essa fibra possui um diâmetro médio elevado (100 a $600 \mu \mathrm{m})$ e como citado em vários trabalhos recentes existe uma correlação inversa entre a variação da resistência à tração e o diâmetro equivalente da FNLs, que é atribuída a maior probabilidade de defeitos internos em maiores diâmetros [2]. Já as fibras de PALF apresentam alto teor de a-celulose e baixo ângulo microfibrilar que resultam na superioridade das suas propriedades mecânicas e um comportamento bem 
distinto da fibra de coco [19].

Com o objetivo de comparar o comportamento dessas duas fibras distintas foram realizados testes de pullout para determinar o comprimento crítico e a adesão interfacial dessas duas fibras lignocelulósicas com a matriz epóxi variando os comprimentos embutidos das fibras conforme proposto por Kelly e Tyson [11].

\section{MATERIAIS E MÉTODOS}

No presente estudo foram usadas as fibras de coco extraídas do mesocarpo do coco verde (Cocos nucifera L.) e as fibras de PALF provenientes das folhas do abacaxizeiro (Ananas comosus). Os perfis típicos dessas fibras estão apresentados na Figura 1. Essas fibras foram usadas na condição como recebida, sem qualquer tratamento superficial, apenas secagem em estufa a $60^{\circ} \mathrm{C}$.

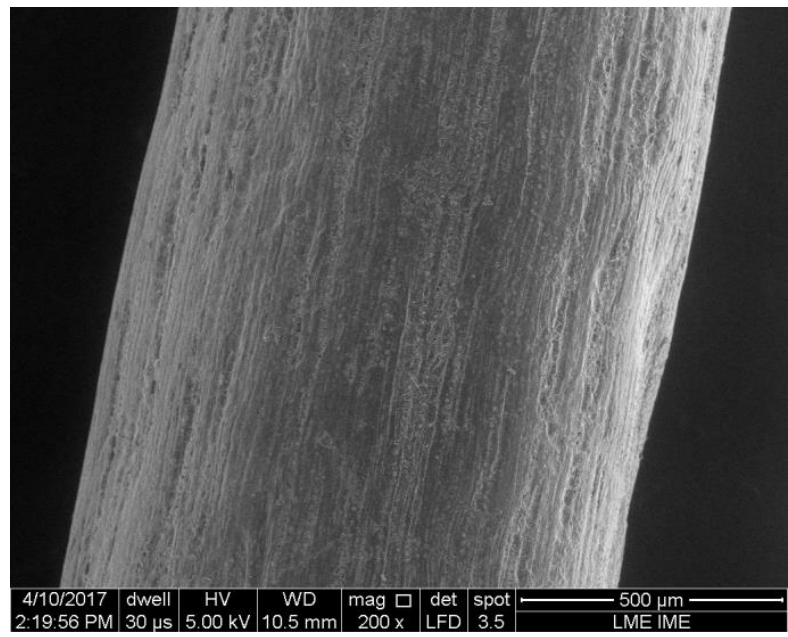

(a)

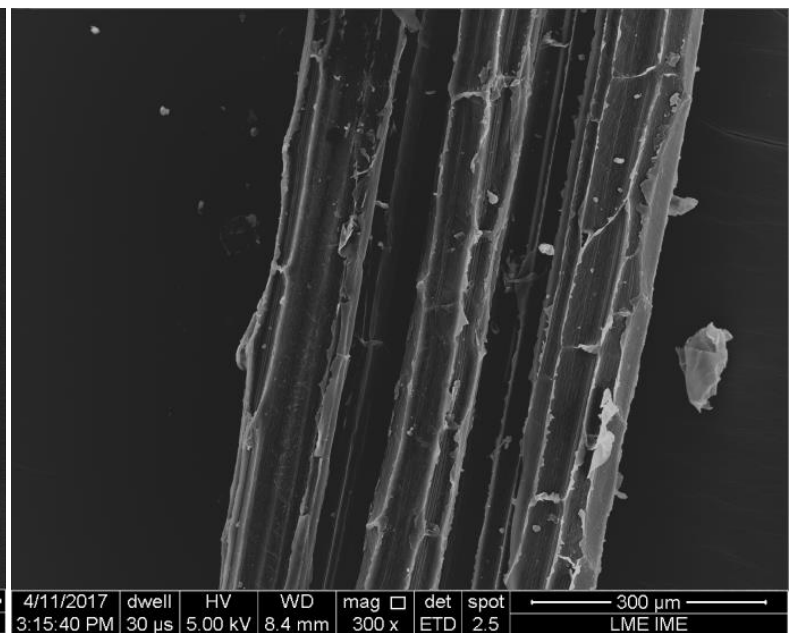

(b)

Figura 1. Imagens por MEV: (a) Perfil da fibra de coco; (b) Perfil do PALF.

Como matriz polimérica foi utilizada a resina epóxi de diglicidil-éter-do-bisfenol-A (DGEBA) e o endurecedor trietilenotetramina (TETA) ambos produzidos pela Dow Chemical do Brasil e fornecidos pela distribuidora Resinpoxy Ltda. Esses dois componentes foram misturados com uma razão estequiométrica phr 13.

Para avaliar a adesão interfacial entre essas fibras e a matriz epoxídica foi realizado o teste pullout, conforme proposto por Kelly e Tyson [11] e adaptado por Monteiro e D'Almeida [12] para fibras lignocelulósicas. Para isso foram preparados blocos cilíndricos de epóxi com diâmetro de $8 \mathrm{~mm}$ variando a profundidade de embutimento de $2,5 \mathrm{~mm}$ a $40 \mathrm{~mm}$ tanto para as fibras de coco quanto para o PALF, como ilustrado esquematicamente na Figura 2.ura 2. O comprimento e o diâmetro das fibras foram medidos com o auxílio de um estereoscópio Zeiss Stemi 2000C.

Os testes de pullout foram realizados em uma máquina universal Instron, modelo 5582, com garras pneumáticas com uma velocidade de $1 \mathrm{~mm} / \mathrm{min}$ e uma pressão de garra de $0,3 \mathrm{MPa}$ foi aplicada nas extremidades da fibra. 


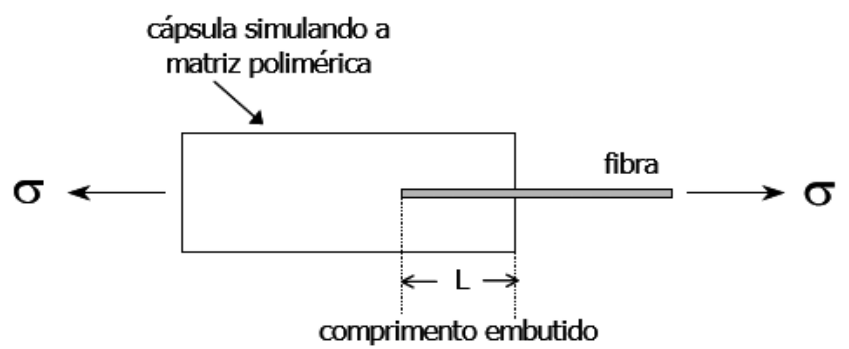

Figura 2. Esquema do corpo de prova para ensaio pullout [13].

\section{RESULTADOS E DISCUSSÃO}

De modo geral, no teste de pullout, a tensão aumenta linearmente com o comprimento embutido da fibra na matriz. Quando essa tensão atinge a tensão de ruptura da fibra ocorre o rompimento da mesma, e o comprimento embutido no qual a fibra falha é denominado de comprimento crítico $\left(\ell_{c}\right)$. Pequenos valores de $\ell_{c}$ indicam maior adesão interfacial fibra/matriz [12]. As características de interface fibra/matriz influenciam diretamente no comportamento mecânico do compósito. É o comprimento crítico que avalia se uma fibra é longa suficiente para agir como reforço de uma dada matriz ou se esta atua apenas como carga, não havendo uma transmissão eficiente de carga aplicada da matriz para a fibra.

A Figura 3 apresenta alguns gráficos de carga versus alongamento obtidos no ensaio de pullout para as diferentes profundidades de embutimento da fibra de coco. Nessa figura observa-se a formação de múltiplos picos devido ao processo de escorregamento gradativo da fibra para os comprimentos embutidos até $20 \mathrm{~mm}$ (Fig. $4 a, b, c)$. Nestes corpos de prova, a fibra foi totalmente arrancada da matriz epóxi sem se romper. Já para os comprimentos de embutimento de 30 e $40 \mathrm{~mm}$ ocorreu a ruptura da fibra antes do desprendimento total da matriz (Fig. 4d, e). Esse mesmo comportamento foi observado para as fibras de PALF.
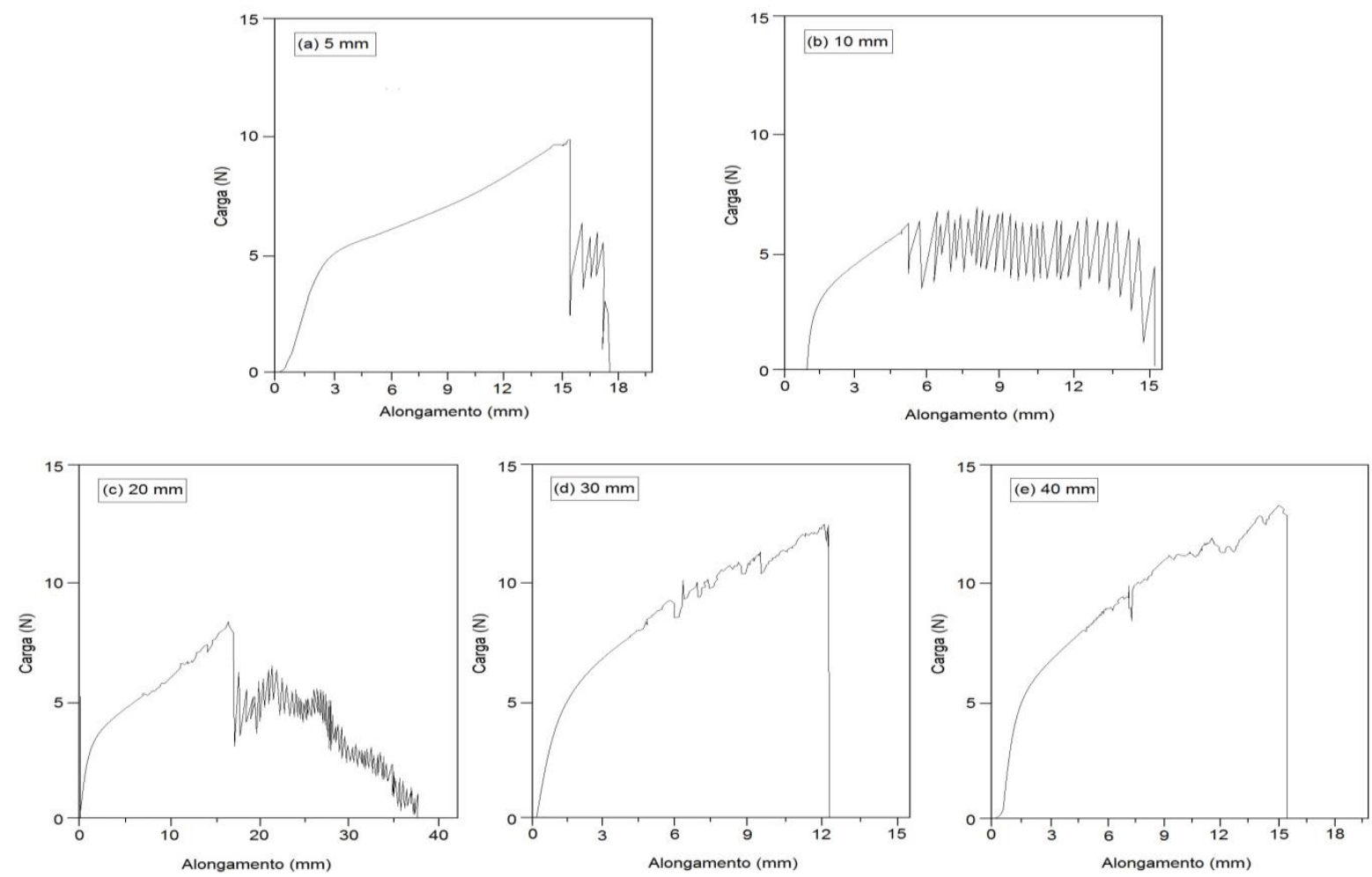

Figura 3. Curva carga versus alongamento para diferentes comprimentos de embutimento da fibra de coco. 
O comprimento crítico da fibra, $\boldsymbol{l}_{c}$, e a resistência interfacial, $\boldsymbol{\tau}_{\boldsymbol{c}}$, das fibras de coco e PALF com a matriz epóxi foram avaliadas por meio da equação de Kelly e Tyson (Equação 1) [11]:

$$
\ell_{c}=\frac{d \cdot \sigma_{f}}{2 \cdot \tau_{c}}
$$

sendo $\sigma_{f}$ a resistência da fibra e $d$ o diâmetro equivalente da fibra.

O valor do diâmetro pode ser uma fonte de erro para as FNLs, pois geralmente suas seções transversais não são circulares e podem variar em formas e dimensões em limites bastante grandes. Para os cálculos realizados neste trabalho, foi determinado o diâmetro equivalente das fibras através da medição da seção transversal ao longo do comprimento de cada fibra. $O$ valor utilizado foi a média de 10 medições, resultando em diâmetros iguais a $520 \mu \mathrm{m}$ e $168 \mu \mathrm{m}$ para as fibras de coco e PALF, respectivamente.

A Figura 4 mostra os resultados do ensaio de pullout para os diferentes comprimentos embutidos (L) das fibras de coco na matriz epoxídica. Pode-se observar que a interseção dos dois primeiros trechos lineares da tensão pullout da fibra, que define o seu comprimento crítico, $\ell_{c}$, é alcançado para $L=12,40 \mathrm{~mm}$ abaixo do qual ocorre o livre desacoplamento da fibra. Como proposto por Monteiro e D'Almeida [12] pode-se definir um segundo comprimento crítico, $L_{c}$, a partir do qual a fibra não desaclopa da matriz e é dado pela interseção da segunda reta com o último trecho linear, obtendo-se um valor $L_{c}=28,75 \mathrm{~mm}$ para a fibra de coco. É importante ressaltar que as barras de erro das tensões associadas aos comprimentos embutidos de 30 a $40 \mathrm{~mm}$ estão dentro do intervalo de resistência a tração de fibras de coco encontrados na literatura [1,2,20,21]. Esse comportamento é esperado e, portanto, apoia os dados experimentais obtidos.

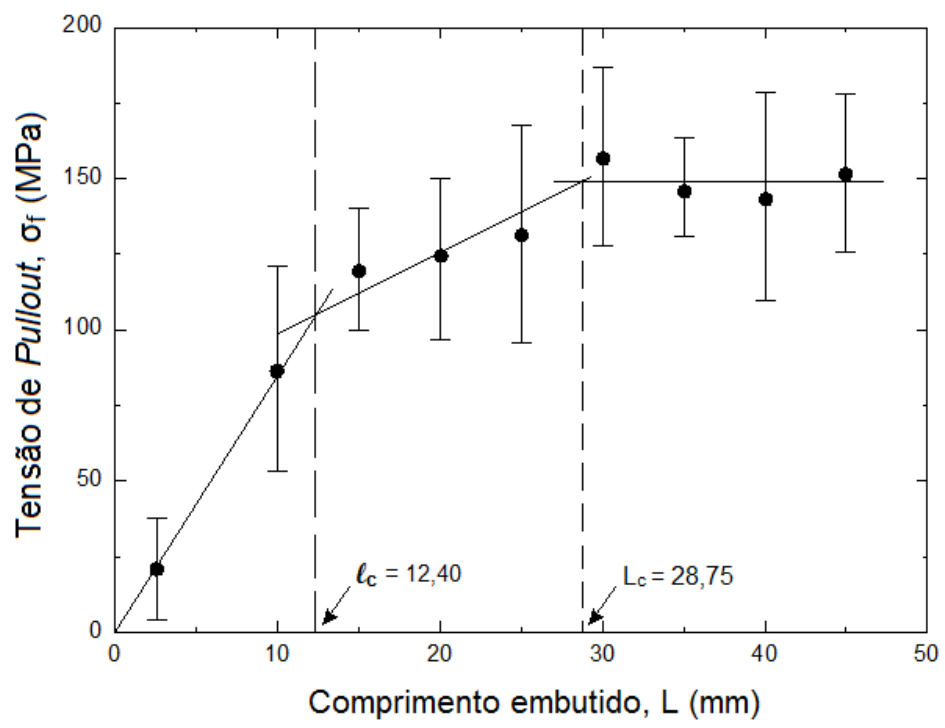

Figura 4. Resultados do ensaio de pullout para os diferentes comprimentos embutidos da fibra de coco na matriz de epóxi.

A Figura 5 mostra a variação da tensão pullout versus o comprimento embutido do PALF. Nota-se que o PALF apresenta uma tensão mais elevada para todos os valores de $\mathrm{L}$ em comparação à fibra de coco, resultando na ruptura da fibra a um comprimento crítico $(7,49 \mathrm{~mm})$ inferior ao da fibra de coco $(12,40 \mathrm{~mm})$, o que indica melhor aderência do PALF na matriz de epóxi. Essa maior adesão pode ser atribuída à superfície naturalmente mais rugosa do PALF [22-25], como pode ser 
observada na Figura 1. Além disso, as maiores tensões obtidas para as fibras de PALF corroboram com a tendência relatada em diversos estudos que indicam que FNLs com menores diâmetros apresentam maior resistência mecânica [2].

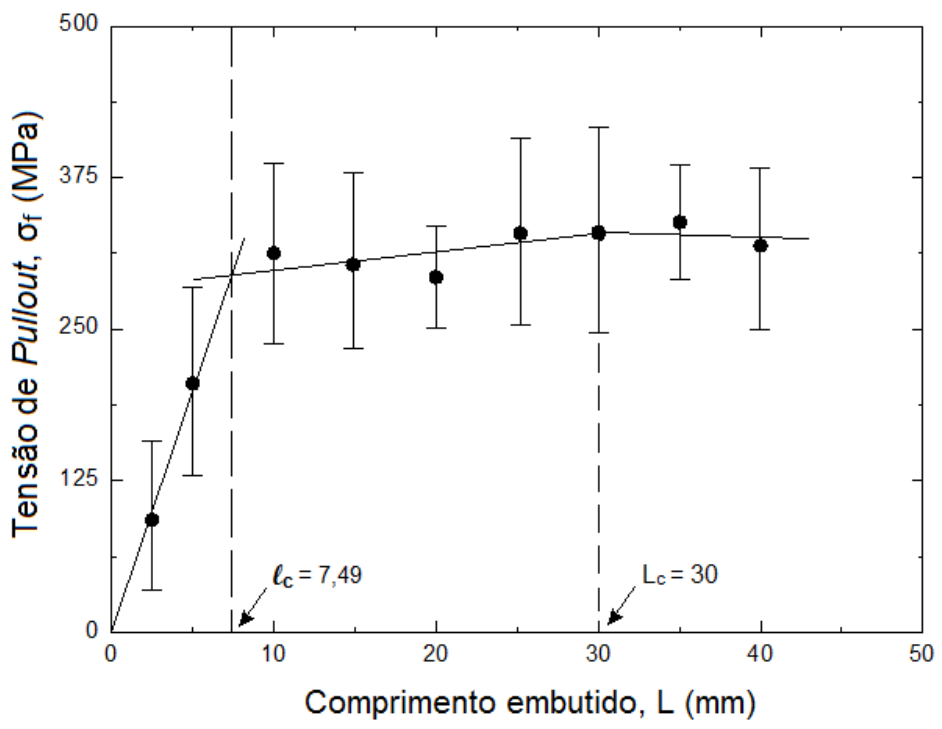

Figura 5. Variação da tensão pullout com o comprimento embutido do PALF na matriz de epóxi.

Com base nos gráficos das Fig. 4 e 5 foram calculadas as tensões cisalhantes na interface para as fibras de coco e PALF, obtendo-se os valores de 2,10 MPa e $3,37 \mathrm{MPa}$, respectivamente. Portanto, a adesão fibra/matriz para a fibra PALF é maior do que para a fibra de coco, isto é, a transferência de tensão da matriz para fibra é mais eficaz para o PALF. Isso sugere uma maior resistência à tração para o compósito de epóxi/PALF. Além disso, comparando esses valores com outras fibras relatadas na literatura $[4,5,12,26]$, pode-se concluir que a interface epóxi/coco é fraca.

\section{CONCLUSÃO}

- Em todos os ensaios de pullout, tanto a fibra de coco quanto o PALF foram totalmente arrancadas da matriz, sem romper, para os comprimentos embutidos abaixo $20 \mathrm{~mm}$;

- Para valores de $\mathrm{L}$ em torno de $30 \mathrm{~mm}$ já não ocorreu mais o desacoplamento da fibra em relação à matriz para essas duas fibras.

- A fibra de coco apresentou um comprimento crítico $65 \%$ maior do que o PALF, o que indica menor adesão interfacial dessa fibra com a matriz epóxi;

- Além disso, a fibra de coco apresentou um diâmetro equivalente três vezes maior que o PALF o que explica as maiores tensões obtidas para as fibras de PALF e corroboram com a tendência relatada em diversos estudos que indicam que FNLs com menores diâmetros apresentam maior resistência mecânica.

\section{Agradecimentos}

Os autores deste trabalho agradecem ao CNPq, à FAPERJ e à CAPES por meio do PDS pelo financiamento da pesquisa, através do programa 31007015006P4. 


\section{REFERÊNCIAS}

1. Faruk O, Bledzki AK, Fink HP, Sain M. Biocomposites reinforced with natural fibers: 2000-2010. Progress in Polymer Science. 2012; 37: 1552-1596.

2. Monteiro SN, Lopes FPD, Barbosa AP, Bavitori AB, Silva ILA, Costal LL. Natural Lignocellulosic Fibers as Engineering Materials - An Overview. Metal. Mater. Trans. A. 2011; 42A: 2963-2974.

3. Monteiro SN, Lopes FPD, Ferreira AS, Nascimento DCO. Natural-Fiber Polymer-Matrix Composites: Cheaper, Tougher, and Environmentally Friendly. Journal of The Minerals, Metals \& Materials Society (JOM). 2009; 61(1):17-22.

4. Aquino RCMP, Monteiro SN, D'Almeida JRM. Evaluation of the critical fiber length of piassava (Attalea funifera) fibers using the pullout test. Journal of Materials Science Letters. 2003; 22(21): 1495-1497.

5. Monteiro SN, Aquino RCMP, Lopes FPD. Performance of curaua fibers in pullout tests. J Mater Sci. 2008; 43(2): 489-492.

6. Bledzki AK, Gassan J. Composites Reinforced with Cellulose Based Fibres. Progress in Polymer Science. 1999; 4: 221-274.

7. Nabi Sahed D, Jog JP. Natural Fiber Polymer composites a review. Advances in Polymer Technology. 1999; 18(4): 351-363.

8. Mohanty AK, Misra M, Hinrichsen G. Biofibres, Biodegradable Polymers and Biocomposites: An Overview. Macromolecular Materials and Engineering. 2000; 276/277: $1-24$.

9. Moura MFSF, Morais AB, Magalhães AG. Materiais Compósitos. Fabrico e Comportamento Mecânico. 2 $2^{\mathrm{a}}$ Ed. Publindustria; 2011.

10. Anderson TL. Fracture mechanics - Fundamentals and Applications. 2nd Ed. New York: CRC Press, 1995.

11. Kelly A, Tyson WR. Tensile properties of fibre-reinforced metals: copper/tungsten and copper/molybdenum. Journal of the Mechanics and Physics of Solids. 1965; 13(6): 329338.

12. Monteiro SN, D’Almeida JRM. Ensaios de Pullout em Fibras Lignocelulósicas - Uma Metodologia de Análise. Revista Matéria. 2006; 11(3): 189-196.

13. Aquino EMF, Sarmento LPS, Oliveira W. Moisture effect on degradation of jute/glass hybrid composites. J. Plast. Comp. 2007; 26: 219-233.

14. Holbery J, Hounston D. Natural-fiber-reinforced polymer composites in automotive applications. JOM. 2006; 58: 80-86.

15. Jayavani S, Deka H, Varghese TO, Nayak SK. Recent Development and Future Trends in Coir Fiber Reinforced Green Polymer Composites: Review and Evaluation. Polym. Compos. 2016; 37(11): 3296-3309.

16. Mir SS, Hasan SMN, Hossain MJ, Hasan M. Chemical modification effect on the mechanical properties of coir fiber. Engineering Journal. 2012; 16(2): 73-84.

17. Wambua $P$, Vangrimde $B$, Lomov $S$, Verpoest I. The response of natural fibre composites to ballistic impact by fragment simulating projectiles. Compos. Struct. 2007; 77: 232-240.

18. Prasad SV, Pavithran C, Rohatgi PK. Alkali treatment of coir fibres for coir-polyester composites. J Mater Sci. 1983; 18: 1443-1454.

19. Satyanarayana KG, Sukumaran K, Mukherjee PS, Pavithran C, Pillai SG. Natural FibrePolymer Composites. Cement \& Concrete Composites. 1990; 12: 117-136.

20. Satyanarayana KG, Fernando W. Characterization of natural fibers. In: Fakirov S, Bhattacharyya $D$ (eds) Engineering biopolymers: homopolymers, blends and composites. Auckland: Hanser, 2007.

21. Kalia S, Kaith BS, Kaurs I. Cellulose Fibers: Bio and Nano-Polymer Composites. 1st Ed. New York: Springer, 2011.

22. Kalia, S, Kaith, B S, Kaur, I. Pretreatments of natural fibers and their application as reinforcing material in polymer composites-a review. Polymer Engineering \& Science. 2009 ; 49(7): 1253-1272. 
23. Ma H, Joo CW. Influence of surface treatments on structural and mechanical properties of bamboo fiber-reinforced poly(lactic acid) biocomposites. Journal of Composite Materials. 2011; 45(23): 2455-2463.

24. Franco PJH, Valadez-González A. Fiber-matrix adhesion in natural fiber composites. In: Natural fibers, biopolymers and biocomposites. Edited by Mohanty AK, Misra M, Drzal LR. Taylor \& Francis, CRC Press, 2005.

25. Rong MZ, Zhang MQ, Liu Y, Yang GC, Zeng HM. The effect of fiber treatment on the mechanical properties of unidirectional sisal-reinforced epoxy composites. Composites Science and Technology. 2001; 61(10): 1437-1447.

26. Brahmakumar M, Pavithran C, Pillai RM. Composites Science and Technology. 2005; 65: 563-569. 